\title{
The Leo-I group: new dwarf galaxy and ultra diffuse galaxy candidates
}

\author{
Oliver Müller ${ }^{1}$, Helmut Jerjen ${ }^{2}$, and Bruno Binggeli ${ }^{1}$ \\ ${ }^{1}$ Departement Physik, Universität Basel, Klingelbergstr. 82, 4056 Basel, Switzerland \\ e-mail: oliver89.mueller@unibas.ch \\ ${ }^{2}$ Research School of Astronomy and Astrophysics, Australian National University, Canberra, ACT 2611, Australia
}

Received 23 February 2018 / Accepted 21 March 2018

\begin{abstract}
Context. The study of dwarf galaxies and their environments provides crucial test beds for predictions of cosmological models and insights into the structure formation on small cosmological scales. In recent years, many problems on the scale of groups of galaxies has challenged the current standard model of cosmology.

Aims. Our aim is to increase the sample of known galaxies in the Leo-I group, which contains the M96 subgroup and the Leo Triplet. This galaxy aggregate is located at the edge of the Local Volume at a mean distance of $10.7 \mathrm{Mpc}$.

Methods. We employed image enhancing techniques to search for low surface brightness objects in publicly available $g r$ images taken by the Sloan Digital Sky Survey within 500 square degrees around the Leo-I group. Once detected, we performed surface photometry and compared their structural parameters to other known dwarf galaxies in the nearby universe.

Results. We found 36 new dwarf galaxy candidates within the search area. Their morphology and structural parameters resemble known dwarfs in other groups. Among the candidates five or six galaxies are considered as ultra diffuse galaxy candidates. If confirmed, they would be some of the closest examples of this galaxy type. We assessed the luminosity function of the Leo-I group and find it to be considerably rich in dwarf galaxies, with twice the number of galaxies as the Local Group at a limiting magnitude of $M_{V}=-10$ and a steeper faint-end slope.
\end{abstract}

Key words. galaxies: groups: individual: Leo-I - galaxies: dwarf - galaxies: photometry galaxies: luminosity function, mass function

\section{Introduction}

More than one thousand galaxies reside in a sphere of $11 \mathrm{Mpc}$ radius around the Milky Way; they are mostly dwarf-type galaxies $\left(M_{B}>-17.7 \mathrm{mag}\right)$. This so-called Local Volume (Kraan-Korteweg \& Tammann 1979; Karachentsev et al. 2004, 2013) contains many prominent galaxy aggregates, e.g., our own Local Group (LG), the Sculptor filament, the Centaurus group, the M81 group, the Canes Venatici cloud, the M 101 group complex, and the Leo-I group (Tully \& Fisher 1988). In recent years many teams have taken up the challenge to search for new dwarf galaxies in the local universe and measure their distances (Chiboucas et al. 2009, 2013; Merritt et al. 2014; Belokurov et al. 2014; Crnojević et al. 2014, 2016; Kim et al. 2015; Müller et al. 2015, 2017a,b; Carlin et al. 2016; Javanmardi et al. 2016; Danieli et al. 2017; Carrillo et al. 2017; Henkel et al. 2017; Park et al. 2017; Makarova et al. 2018). These studies can be used to test the theoretical predictions from the standard model of cosmology $(\Lambda \mathrm{CDM})$. For the $\mathrm{LG}$, there is a serious tension between observation and theory represented by the long-standing missing satellite problem (Moore et al. 1999), the too-big-too-fail (TBTF) problem (Kroupa et al. 2010; Boylan-Kolchin et al. 2011), and the plane-of-satellites problem (Kroupa et al. 2005; Pawlowski et al. 2012; Ibata et al. 2013; Pawlowski 2018), see Bullock \& Boylan-Kolchin (2017) for a recent review on small-scale challenges. Such studies are now extended to other nearby galaxy groups, for example to address the plane-of-satellite problem in Cen A (Tully et al. 2015; Müller et al. 2016, 2018) or the TBTF and missing satellite problems in M 101 (Danieli et al. 2017; Müller et al. 2017b).

Using public data from the Sloan Digital Sky Survey (SDSS) we have started to systematically search for new or hitherto undetected dwarf galaxies in the Local Volume, beginning with the M 101 group complex, covering $330 \mathrm{deg}^{2}$ around the spiral galaxies M 101, M 51, and M 63. We found 15 new dwarf galaxy candidates (Müller et al. 2017b). We now continue our optical search for dwarf galaxies in an area that covers $500 \mathrm{deg}^{2}$ around the Leo-I group (Fig. 1).

The Leo-I group, with a mean distance of $10.7 \mathrm{Mpc}$ (Karachentsev et al. 2004, 2013), consists of seven bright galaxies, NGC 3351 (=M 95), NGC 3368 (=M 96), NGC 3377, NGC 3379 (=M 105), NGC 3384, NGC 3412, and NGC 3489 (Karachentsev \& Karachentseva 2004). Another four bright galaxies, NGC 3632 (=M 65), NGC 3627 (=M 66), NGC 3628 (which make up the Leo Triplet, about six degrees to the east of the main aggregate), and NGC 3593, are possibly also part of the group based on their common distances and systemic velocities (Ferrarese et al. 2000). We note that about eight degrees to the northeast is another quartet of bright galaxies (NGC 3599, NGC 3605, NGC 3607, and NGC 3608), which shares the same systemic velocity but is farther behind and is arguably not associated with the group (Ferrarese et al. 2000).

A spectacular feature of the Leo-I group in HI is known as the Leo ring (Schneider 1985) around NGC 3384/M 105, one of the largest HI structures in the nearby universe. Michel-Dansac et al. (2010) followed this up with a deep optical 


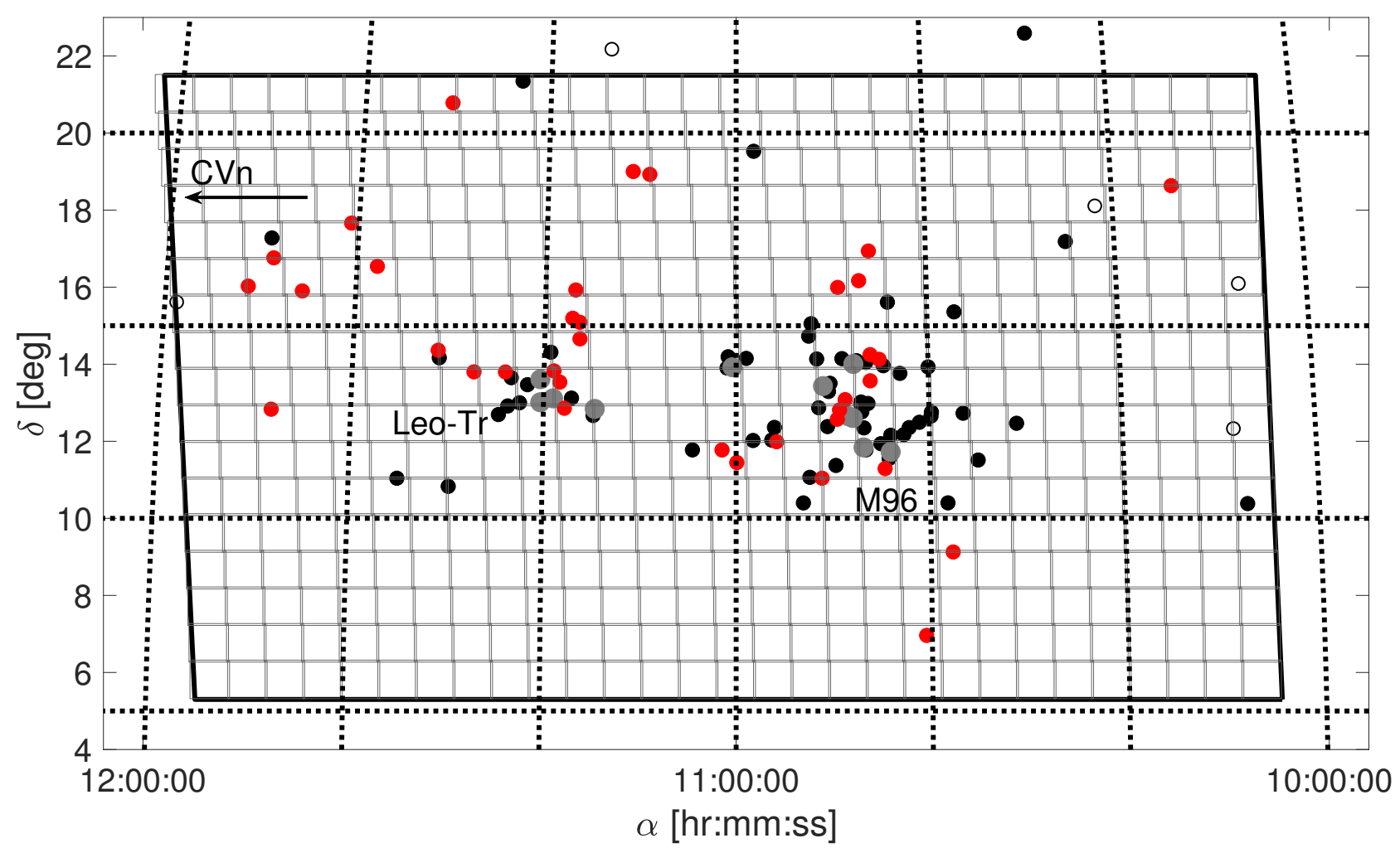

Fig. 1. Survey area of $\approx 500 \mathrm{deg}^{2}$ in the Leo-I group region. The squares correspond to the created $1 \mathrm{deg}^{2}$ mosaics. The small black dots are previously known members based on their photometric properties, compiled from the Local Volume Catalog (Karachentsev et al. 2004, 2013). The large gray dots are the major galaxies in the M96 subgroup and Leo Triplet. The red dots indicate the positions of the 36 new dwarf candidates. Open circles are confirmed foreground galaxies $(<7 \mathrm{Mpc})$ taken from the LV Catalog.

survey using MegaCam on the CFHT and found no diffuse stellar optical component down to $28 \mathrm{mag} \operatorname{arcsec}^{-2}$ surface brightness. The authors suggest an origin based on a collision between NGC 3384 and M 105 using gas and dark matter simulations that can explain the structure of the ring, together with the absence of apparent light. Deeper images $\left(\mu_{V}>29.5 \mathrm{mag} \operatorname{arcsec}^{-2}\right)$ taken by Watkins et al. (2014) have not yet revealed no optical counterpart of the ring; however, they found some stream-like features associated with the ring that are possibly of tidal origin. In the Leo Triplet another intriguing feature, this time in the optical, is a stellar stream associated with the boxy spiral NGC 3628 (Zwicky 1956), which hosts a tidal dwarf galaxy (Nikiel-Wroczyński et al. 2014) and an ultra compact dwarf galaxy (Jennings et al. 2015).

For the central part of the Leo-I group (i.e., the M96 subgroup) an initial catalog of 50 dwarf galaxy candidates was produced by Ferguson \& Sandage (1990). The authors argued, based on morphological properties, that half of them are group members. Another collection of dwarf galaxies was discovered by Trentham \& Tully (2002) who surveyed a $10 \times 10 \mathrm{deg}^{2}$ field partially covering the Leo-I group. Using the digitized sky survey, Karachentsev \& Karachentseva (2004) refined and extended this list to 50 likely members. For many members HI velocities were derived (Stierwalt et al. 2009), making it possible to distinguish between actual Leo-I members and background galaxies belonging to the more distant Leo cloud (see Fig. 1 in Trentham \& Tully 2002 for the difference in velocity space). A very deep but spatially limited image, based on amateur telescopes, was produced for NGC 3628 in the Leo Triplet and revealed another faint dwarf galaxy (Javanmardi et al. 2016).

To follow a consistent naming convention in this paper, from now on we use the term M96 subgroup to describe the main galaxy aggregate around M96, and the term Leo-Triplet (Leo-Tr) for the aggregate around M 66. Both subgroups together are called the Leo-I group (see Fig. 1).

In this work we present a search for unresolved dwarf galaxies using publicly available data from the Sloan Digital Sky Survey (SDSS) in $500 \mathrm{deg}^{2}$, covering the extended Leo-I group region. In Sect. 2 we summarize our search strategy, and in Sect. 3 we present the surface photometry performed for all known and newly found members of the Leo-I group. In Sect. 4 we discuss our candidate list and potential background contamination. Finally, in Sect. 5 we draw our conclusions and give a brief outlook.

\section{Discovery of new dwarf galaxy candidates}

In recent years, different automatic detection approaches have been proposed to search for low surface brightness galaxies (e.g., Merritt et al. 2014; Speller \& Taylor 2014; van der Burg et al. 2016; Bennet et al. 2017) with encouraging results. On the other hands, these pipelines were only applied on small areas of the sky $\left(<10 \mathrm{deg}^{2}\right)$ and still have a considerable rate of false detections, or rely on a large number of existing galaxies to study galaxy groups on a statistical basis. It remains to be seen how these methods perform on large-field surveys with areas of several hundred of square degrees and how time-consuming the task of rejecting false-positives will be. We argue, as do other authors (e.g., Park et al. 2017; Wittmann et al. 2017), that a visual search on images is still on par with algorithm-based detections.

In this work, we follow the same methods as described in Müller et al. (2017b) to search for dwarf galaxies in an area 




Fig. 2. Gallery showing SDSS $r$-band images of the new Leo-I group member candidates. One side of an image corresponds to 80 arcsec or $3.88 \mathrm{kpc}$ at the distance of $10 \mathrm{Mpc}$. North is to the top, east to the right.

of $\sim 500 \mathrm{deg}^{2}$ around the Leo-I group using data taken from the SDSS. In summary this involves the creation of 1 square degree mosaics of $g$ and $r$ images, the use of several image processing algorithms (e.g., binning and Gaussian convolution) to enhance the low surface brightness features within the images, and the final visual search for dwarf galaxies in these processed images. Once an object is detected, surface photometry is applied to derive the structural parameters, which are compared to the properties of known dwarf galaxies of the LG and other groups. Based on this morphological comparison, a detection is considered or rejected as a dwarf galaxy candidate. To estimate our detection rate we conducted an experiment where we induced artificial galaxies into the SDSS images and derived the recovery rate of these objects (Fig. 3 in Müller et al. 2017b).

In Fig. 1 we present the survey footprint, the known galaxies in this field (black and gray dots), and the new dwarf galaxy candidates (red dots) found in our search. In the up-to-date online version $^{1}$ of the $\mathrm{LV}$ catalog, 63 dwarf galaxies are listed within our footprint, with four (open circle) having a distance estimate smaller than $7 \mathrm{Mpc}$. In Table A.1 we present the coordinates of the 36 dwarf galaxy candidates found in the survey, together with our galaxy type classification and comments on the objects. We indicate whether the objects are found in the vicinity of M96, in

last checked: 19 December 2017. the Leo Triplet, or in the surrounding field. In Fig. 2 we present images of the newly discovered candidates. Some dwarf galaxy candidates show irregularities. We checked the Galaxy Evolution Explorer (GALEX) survey (in the near- and far-ultraviolet) for extended objects coinciding with our detections. Indeed, the candidates dw1013+18, dw1045+14a, dw1049+15, dw1116+15b, $\mathrm{dw} 1130+20$, and $\mathrm{dw} 1148+16$ have some UV features, possibly hinting towards star formation. These objects are classified as dIrr/dSph if they possess a smooth profile and are likely transition type dwarfs.

\section{Surface photometry}

We computed the total apparent magnitude $m$, the mean effective surface brightness $\langle\mu\rangle_{\mathrm{eff}}$, and the effective radius $r_{\mathrm{eff}}$ in $g r$ bands for each dwarf galaxy candidate, and for already known group members as many of them do not have accurate photometry. To measure the surface brightness profiles we used a circular aperture (step size of 0.'396 corresponding to 1 pixel). Sérsic profiles (Sersic 1968) were fitted at the derived profiles using the equation

$\mu_{\text {sersic }}(r)=\mu_{0}+1.0857 \times\left(\frac{r}{r_{0}}\right)^{n}$, 

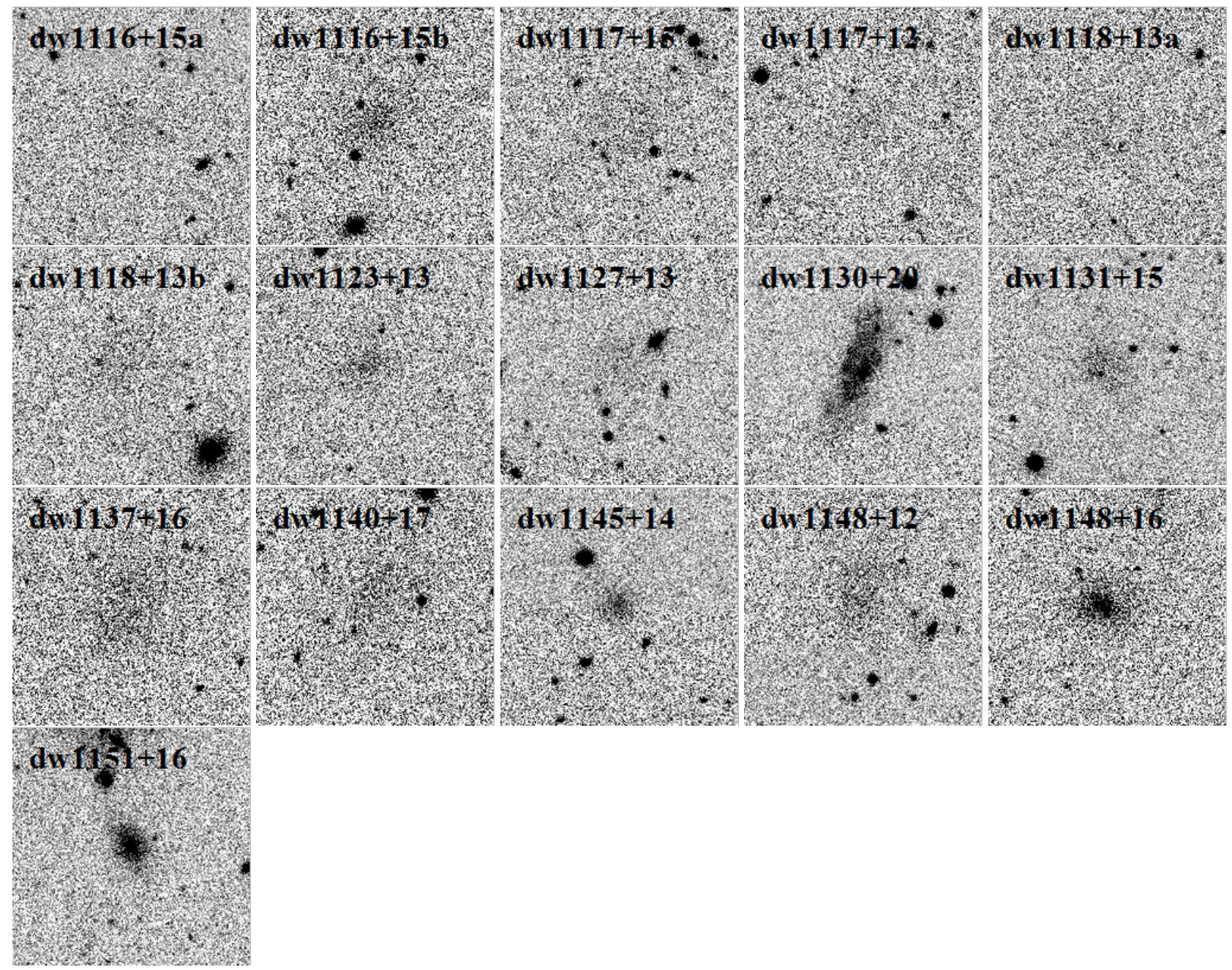

Fig. 2. continued.

where $\mu_{0}$ is the Sérsic central surface brightness, $r_{0}$ the Sérsic scale length, and $n$ the Sérsic curvature index. The total extinction corrected absolute magnitude $M$ is calculated with a distance modulus of $m-M=30.06 \mathrm{mag}$, corresponding to $D=10.4 \mathrm{Mpc}$, as is used for Leo-I members with unknown distance estimates in the LV catalog (see Fig. 3 for all surface brightness profiles in the $r$ band and the associated Sérsic fits). In Table A. 2 we provide the derived photometry for the new candidates, and in Tables A.3 and A.4 the previously known (dwarf) members of the Leo-I group.

The magnitude uncertainties are estimated at around $\approx 0.3$ mag (Müller et al. 2017b). The main contributions to the error budget are from the uncertainties related to foreground star removal $(\approx 0.2 \mathrm{mag})$ and sky background estimation $(\approx 0.2 \mathrm{mag})$. The uncertainties for $\langle\mu\rangle_{\text {eff }}$ are driven by the uncertainties in the measured total apparent magnitude $\left(\approx 0.3 \mathrm{mag} \operatorname{arcsec}^{-2}\right)$. The error for $r_{\text {eff }}(\approx 1.3 \mathrm{arcsec})$ is given by the determination of the growth curve. Numerical uncertainties for the Sérsic parameters are provided in the corresponding table.

\section{Discussion}

In the following we discuss the membership of the candidates based on their morphological parameters, the contamination of the field by nearby background galaxies, and the potential discovery of ultradiffuse galaxies (UDG).

\subsection{Membership estimation}

The standard approach for establishing membership based on morphological properties is to compare the structural parameters of the candidates with known dwarf galaxies (e.g., Jerjen et al. 2000; Chiboucas et al. 2009; Merritt et al. 2014; Müller et al. 2017a,b). If the objects fit into the $\left(\langle\mu\rangle_{\mathrm{eff}}-M\right),\left(r_{\mathrm{eff}}-M\right)$, $\left(\mu_{0}-M\right)$, and $(n-M)$ scaling relations defined by the known dwarf galaxies in the local Universe, it is reasonable to consider them as dwarf galaxy candidates. The $\left(\langle\mu\rangle_{\mathrm{eff}}-M\right)$ and $\left(\mu_{0}-M\right)$ are especially crucial because the surface brightness is independent of the assumed distance of the object, therefore making it possible to assess the membership at a certain distance (see Müller et al. 2017a, Fig. 11 for what happens to galaxies with unreasonable distance estimates in those relations). To transform our $g r$ photometry to the Johnson system we used the following equations (Lupton 2005):

$V=g-0.5784 \times(g-r)_{0}-0.0038$.

$B=r+1.3130 \times(g-r)_{0}+0.2271$.

The structural parameters of the newly found dwarf candidates, and of the previously discovered Leo-I members and the Local Group dwarf population, are plotted in Fig. 4. The structural parameters of the dwarf candidates fall into the 

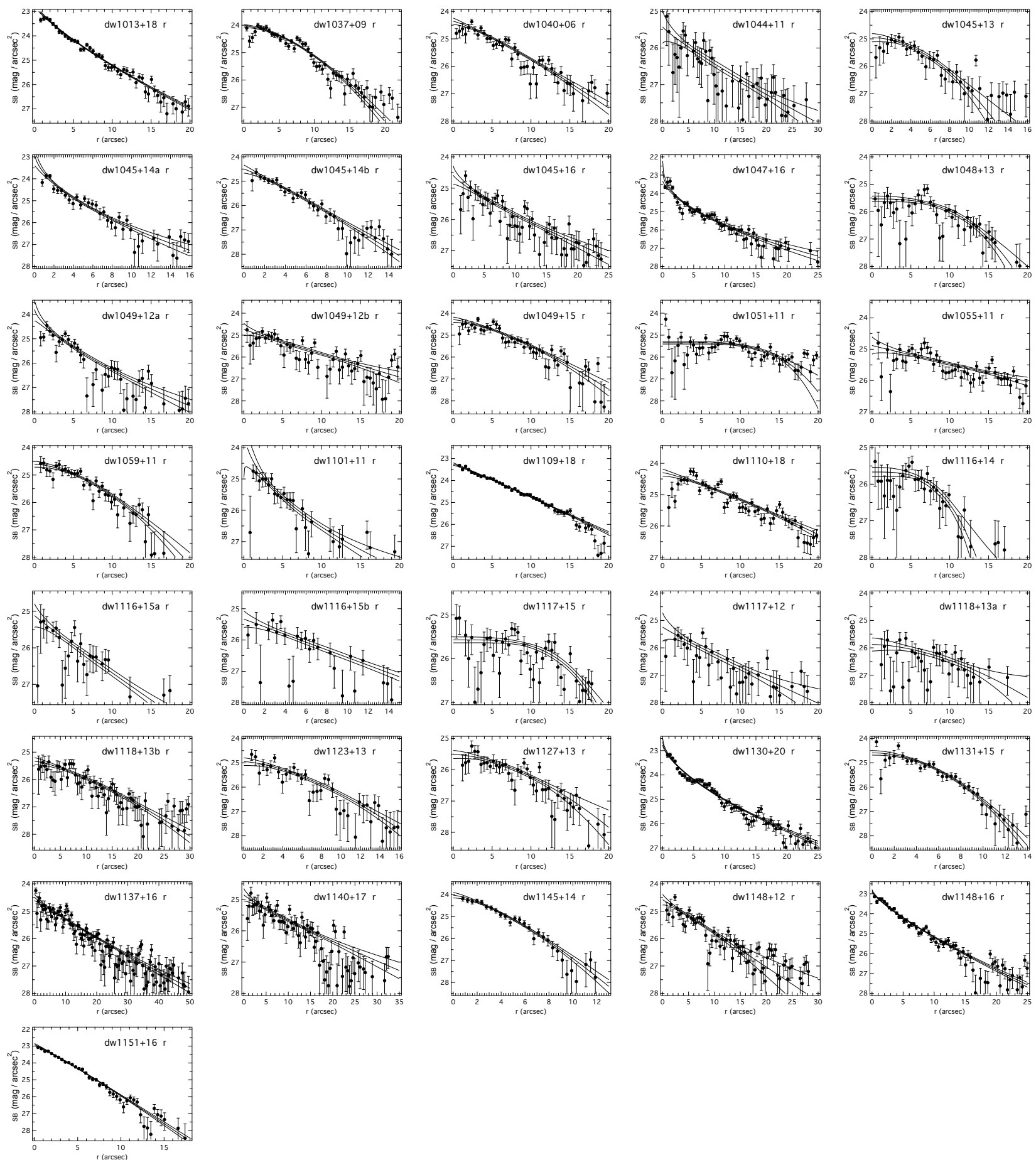

Fig. 3. Surface brightness profiles of all new dwarf galaxy candidates in $r$ and the best-fitting Sérsic profiles with $1 \sigma$ confidence intervals.

relations defined by the Local Group dwarfs, thus we can assume that the candidates are indeed dwarf members of the Leo-I group. Additionally, we show the 44 UDG candidates in the Coma Cluster discovered by van Dokkum et al. (2015), who only gave $g$ band photometry and so we assume a color index of $(g-r)=0.6 \mathrm{mag}$ to transform them into $V$-band magnitudes. UDGs typically have an effective radius larger than $r_{\mathrm{eff}}>1.5 \mathrm{kpc}$ and a central surface brightness fainter than $\mu_{g}>24.0$ mag $\operatorname{arcsec}^{-2}$ (van Dokkum et al. 2015).

Dwarf galaxies can also be characterized by their color using the color-magnitude relation (e.g., Lisker et al. 2008; Venhola et al. 2017). Here we compare the $(g-r)_{0}$ colors of the Leo-I group dwarfs with other well-studied systems in the LV where gr photometry is available, namely the Centaurus group (Müller et al. 2015, 2017a) and the M101 group complex (Müller et al. $2017 b)$. The calculated mean $(g-r)_{0}$ color and standard deviation for the three group populations are $(g-r)_{0, \text { Leo }-I}=0.491 \pm$ $0.282 \mathrm{mag},(g-r)_{0, \mathrm{Cen} A}=0.463 \pm 0.258 \mathrm{mag}$, and $(g-r)_{0, M 101}=$ $0.472 \pm 0.190$ mag. In Fig. 5 we show the color distribution as a function of total absolute $V$-magnitude for these different groups. The dwarfs in the different galaxy groups follow a similar distribution in their colors. We note that the extreme blue 

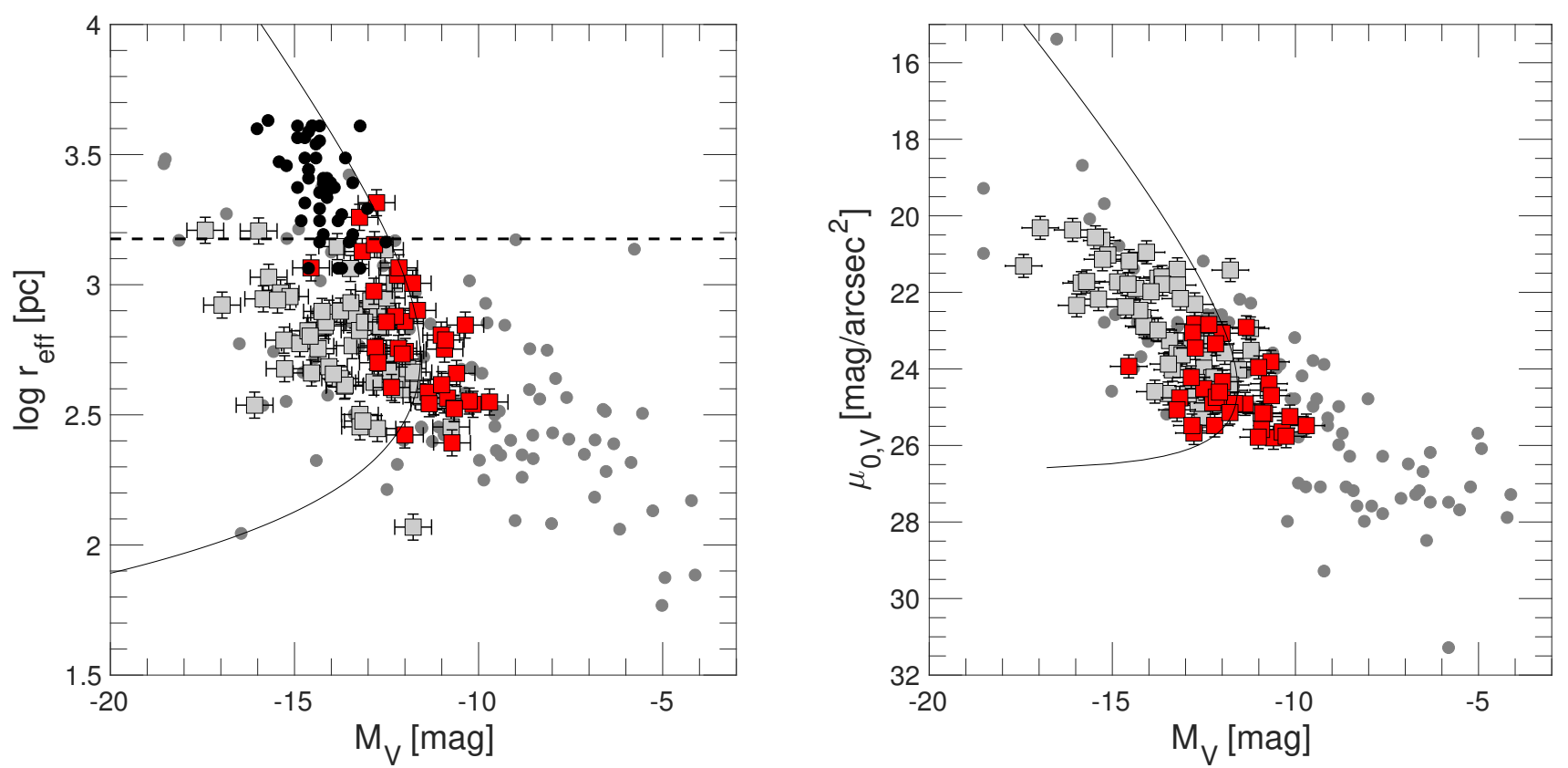

Fig. 4. Scaling relations $\left(r_{\mathrm{eff}}-M\right)$ and $\left(\mu_{0}-M\right)$ for the newly discovered dwarf candidates (red squares), previously discovered dwarf members (gray squares), and the Local Group dwarf galaxy population (gray dots). The estimated conservative completeness limit, as derived in Müller et al. (2017b), is indicated with the line. The UDG candidates discovered in Coma (van Dokkum et al. 2015) are overlaid as black dots in the ( $r_{\text {eff }}-M$ ) diagram; also overlaid is the size cut (dashed line) of $1.5 \mathrm{kpc}$ for UDGs.

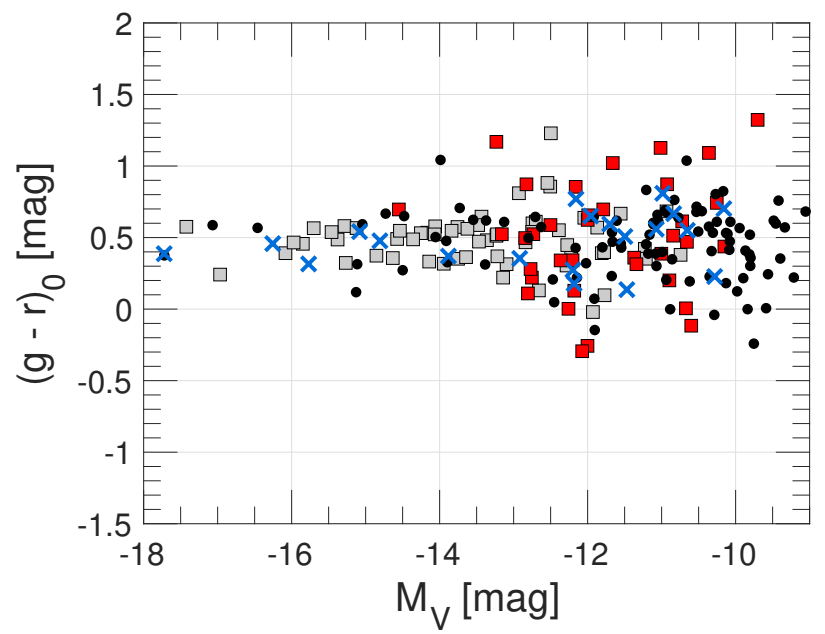

Fig. 5. Color-magnitude relation for the previously known Leo-I dwarf members (gray squares), the new Leo-I members (red squares), the Centaurus group members (black dots, Müller et al. 2015, 2017a), and the M 101 group members (blue crosses, Müller et al. 2017b). Both early- and late-type dwarf galaxies were considered.

colors $(g-r<0)$ of some objects, which is uncommon for dwarf galaxies, and the scatter at the faint-end of the scale can arise from the photometric uncertainty.

In the following we discuss some individual candidates that have interesting features.

$d w 1037+09$. This candidate has several knots within and around the galaxy, which could either be bright giant stars or globular clusters (GC).

$d w 1110+18$. Here too there are several knots sprinkled through the object, which could be bright giant stars or GCs.

$d w 1130+20$. This galaxy has some bright knots, which could correspond to HII regions.

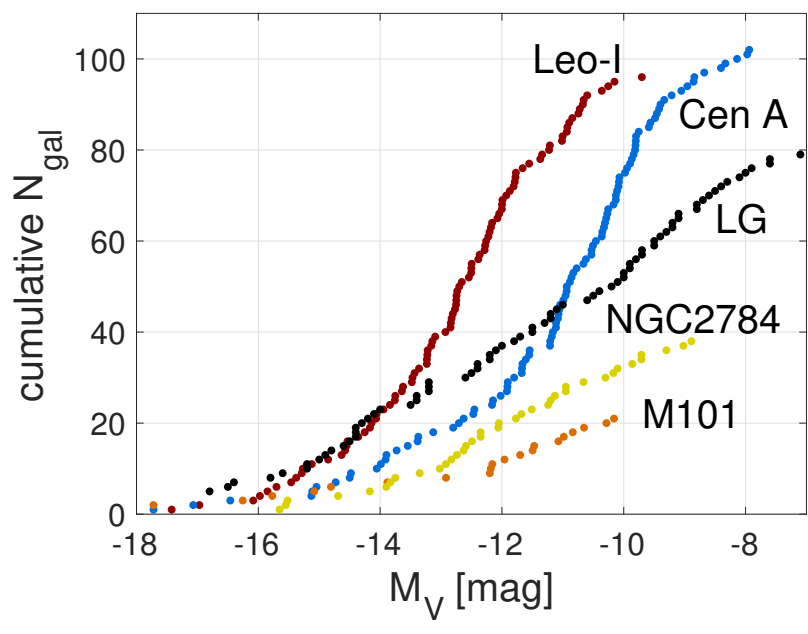

Fig. 6. Cumulative galaxy luminosity functions for different galaxy groups in the Local Volume. Data taken from Leo-I (this work), Centaurus group (Müller et al. 2015, 2017a), LG (McConnachie 2012), NGC 2784 group (Park et al. 2017), and M 101 group (Bremnes et al. 1999; Müller et al. 2017b).

Under the assumption that all candidates are members of the Leo-I group, we can determine the galaxy luminosity function (see Fig. 6) and compare it to other nearby galaxy group environments, i.e., the Centaurus group (Müller et al. 2015, 2017a), the LG (McConnachie 2012), the M101 group (Bremnes et al. 1999; Müller et al. 2017b), and the NGC2784 group (Park et al. 2017). Among these five groups, the Leo-I group is the richest galaxy aggregate with approximately 100 galaxies up to an absolute magnitude of $M_{V}=-10$, in other words if all candidates are confirmed as members. The Leo-I group has approximately twice as many dwarfs as the LG and a steeper faint-end slope of the LF, comparable to that of Cen A. The 


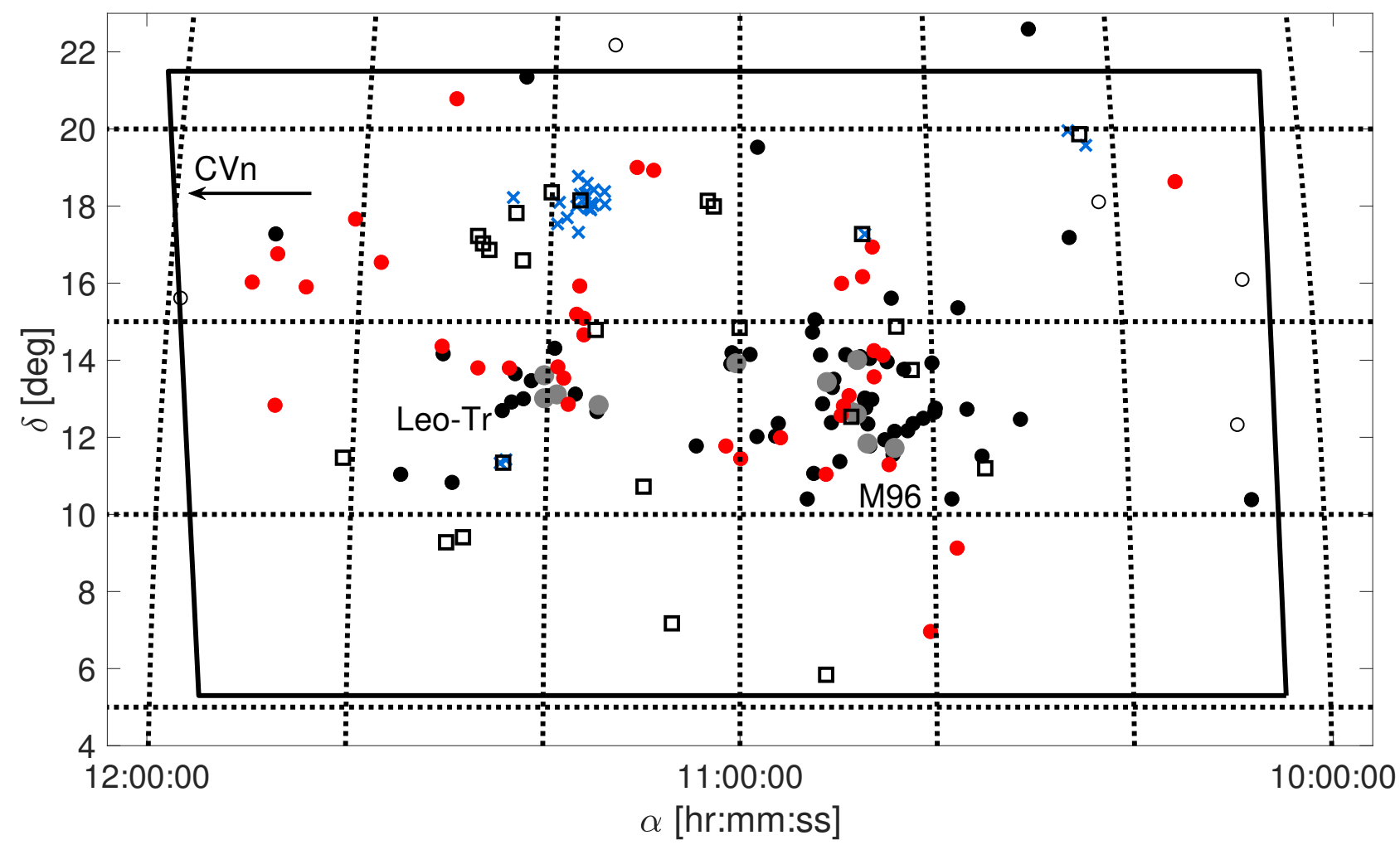

Fig. 7. Same as Fig. 1, but with background host galaxies (black squares) and the background dwarf galaxies (blue crosses) that are clustered around these hosts. The background dwarfs would be considered Leo-I dwarfs (thanks to their morphology) if they were not so close to the background hosts. See Sect. 4.2 for details.

M 101 and NGC 2784 groups have shallower faint-end slopes. This indicates that galaxy groups with massive hosts have steeper faint-ends of the LF. While the faint-end slopes of Leo-I and Cen A are comparable, the Leo-I group contains more brighter galaxies in the range from -16 to -14 mag in $V$-bands, making it more rich (up to $\left.M_{V}=-10\right)$. In this range ( -16 to $-14 \mathrm{~V}$ mag), the LF of Leo-I is comparable to that of the LG.

\subsection{Background contamination}

One fundamental challenge when searching for new dwarf galaxies is that survey fields are almost always contaminated by galaxy groups in the background. A prime example for such a confusion is the massive elliptical galaxy NGC 5485 with its many dwarf companions (Makarov \& Karachentsev 2011) situated $\approx 20 \mathrm{Mpc}$ behind the Local Volume galaxy M 101 (7 Mpc, Nataf 2015). Figure 8 in Merritt et al. (2016) shows M 101, the background elliptical NGC 5485, and former M 101 dwarf candidates (Merritt et al. 2014) that actually belong to the background galaxy population. Out of the seven dwarf candidates reported by Merritt et al. (2014), only three were confirmed to be M 101 members with HST follow-up observations (Danieli et al. 2017). Recently, more new dwarf candidates were reported around M 101 (Bennet et al. 2017; Müller et al. 2017b), now awaiting confirmation as members by means of distance or velocity measurements. Some will potentially be associated with the background elliptical NGC 5485.

The possibility of contamination prompted us to study the background of the Leo-I group in more detail. In Müller et al. (2017a) we used the Cosmicflows-2 catalog (Tully et al. 2013) to determine the background contamination of the Centaurus group. Here we query the Cosmicflows- 2 catalog for bright galaxies with absolute magnitudes $M_{B}<-19$ and with radial velocities $v_{\text {rad }}<2000 \mathrm{~km} \mathrm{~s}^{-1}$ within our survey footprint. Excluding the Leo-I galaxies this search resulted in 24 bright host galaxies potentially contaminating our survey.

To test how these background galaxies will pollute our detections we surveyed for dwarf galaxies within $300 \mathrm{kpc}$ of each such host (approximately the virial radius) with the same methods as used in our search for Leo-I dwarfs, but without removing candidates that are near a background galaxy. Essentially, we search for the candidates we rejected as background sources. In Table A. 3 we compiled the coordinates for the objects that would be considered as dwarf candidates based on their morphology. In total we found 26 additional dwarf candidates, of which 20 are clustered around NGC 3607 at a distance of $\sim 20 \mathrm{Mpc}$. This indicates that (a) it is not feasible to include every object in the survey footprint as Leo-I dwarf, and (b) that there will probably be some confusion between foreground and background, either by rejecting a foreground dwarf or including a background dwarf.

Some Leo-I dwarf candidates are near both a background host and a Leo-I host. In this case we added a note to Table A.1. To the north to the Leo Triplet there are four Leo-I candidates (dw1116+14, dw1116+15a, dw1116+15b, and dw1117+15) clustered around NGC $3596(15 \mathrm{Mpc})$. The distribution of the background dwarf galaxies can be seen in Fig. 7. Distance and velocity measurements will be crucial to distinguish their memberships. Until then, the faint-end of the LF will be affected by these uncertain cases.

\subsection{UDG candidates}

Originally discovered by Sandage \& Binggeli (1984) and described as "a new type of very large diameter (10000 pc), 
low central surface brightness ( $>25 \mathrm{~B}$ mag $\operatorname{arcsec}^{-2}$ ) galaxy, that comes in both early (i.e., $\mathrm{dE}$ ) and late (i.e., $\mathrm{Im} \mathrm{V)}$ types," this class of galaxies is now called ultradiffuse galaxies (van Dokkum et al. 2015); these galaxies have been found in many different environments (van der Burg et al. 2016), for example in clusters (van Dokkum et al. 2015; Koda et al. 2015), and in groups (Merritt et al. 2016). Different possible formation scenarios have been proposed (e.g., Amorisco \& Loeb 2016; Di Cintio et al. 2017) and are under intense debate. van Dokkum et al. (2015) suggested classifying dwarf galaxies with $r_{\text {eff }}>1.5 \mathrm{kpc}$ and a fainter central surface brightness than $\mu_{g}>24.0$ mag $\operatorname{arcsec}^{-2}$ as UDGs; however, this boundary is rather arbitrary and should be considered more as a guideline.

Studying the properties of the Leo-I members we consider dw1055+11, dw1117+15, dw1051+11, KK 96, and ACG 215415 as UDG candidates. With $r_{\text {eff }}=1.3 \mathrm{kpc} \mathrm{dw} 1137+16$ is still considerably large and could be a UDG type. Better photometry is needed to derive the structural parameters more accurately. However, we note that if these objects were more in the foreground (e.g., in the Canes Venatici-I cloud), they would be closer to our point of view and therefore would have smaller intrinsic sizes, making them common-sized dwarf galaxies.

The UDG candidates are distributed in the outskirts of the aggregates and not in the central parts of the group. This is similar to what is found in galaxy clusters: in galaxy clusters the UDG density drops nearly to zero in the central regions because they cannot survive the tidal forces inflicted on them (van der Burg et al. 2016). We note that it is not feasible to assess the UDG distribution in Leo-I with only 5 or 6 candidates.

\section{Conclusion}

We have surveyed 500 square degrees of $g r$ images taken from SDSS within the extended region of the Leo-I group and found 36 new dwarf galaxy candidates. For every known member and new candidate we derived surface brightness photometry. Based on a comparison of their structural properties with other known dwarf galaxies in the nearby universe and their morphology we consider these candidates to be members of the Leo-I group, lying in the vicinity of the M96 subgroup, in the Leo Triplet, or in the nearby field. To confirm their membership, follow-ups are required to either measure their radial velocities, their distances, or both. Some of the candidates are exceptionally large with low surface brightness, a characteristic of ultradiffuse galaxies. If these UDGs are confirmed as Leo-I members, they would be some of the closest UDGs to Earth and valuable targets that could be used to improve our understanding of this galaxy type.

Acknowledgements. $\mathrm{OM}$ and $\mathrm{BB}$ are grateful to the Swiss National Science Foundation for financial support. HJ acknowledges the support of the Australian Research Council through Discovery Project DP150100862. We thank the referee for the helpful comments.

\section{References}

Amorisco, N. C., \& Loeb, A. 2016, MNRAS, 459, L51

Belokurov, V., Irwin, M. J., Koposov, S. E., et al. 2014, MNRAS, 441, 2124 Bennet, P., Sand, D. J., Crnojević, D., et al. 2017, ApJ, 850, 109 Boylan-Kolchin, M., Bullock, J. S., \& Kaplinghat, M. 2011, MNRAS, 415, L40 Bremnes, T., Binggeli, B., \& Prugniel, P. 1999, A\&AS, 137, 337

Bullock, J. S., \& Boylan-Kolchin, M. 2017, ARA\&A, 55, 343

Carlin, J. L., Sand, D. J., Price, P., et al. 2016, ApJ, 828, L5

Carrillo, A., Bell, E. F., Bailin, J., et al. 2017, MNRAS, 465, 5026
Chiboucas, K., Karachentsev, I. D., \& Tully, R. B. 2009, AJ, 137, 3009 Chiboucas, K., Jacobs, B. A., Tully, R. B., \& Karachentsev, I. D. 2013, AJ, 146, 126

Crnojević, D., Sand, D. J., Caldwell, N., et al. 2014, ApJ, 795, L35

Crnojević, D., Sand, D. J., Spekkens, K., et al. 2016, ApJ, 823, 19

Danieli, S., van Dokkum, P., Merritt, A., et al. 2017, ApJ, 837, 136

Di Cintio, A., Brook, C. B., Dutton, A. A., et al. 2017, MNRAS, 466, L1

Ferguson, H. C., \& Sandage, A. 1990, AJ, 100, 1

Ferrarese, L., Ford, H. C., Huchra, J., et al. 2000, ApJS, 128, 431

Haynes, M. P., Giovanelli, R., Martin, A. M., et al. 2011, AJ, 142, 170

Henkel, C., Javanmardi, B., Martínez-Delgado, D., Kroupa, P., \& Teuwen, K. 2017, A\&A, 603, A18

Huchtmeier, W. K., Karachentsev, I. D., \& Karachentseva, V. E. 2003, A\&A, 401,483

Ibata, R. A., Lewis, G. F., Conn, A. R., et al. 2013, Nature, 493, 62

Javanmardi, B., Martinez-Delgado, D., Kroupa, P., et al. 2016, A\&A, 588, A89

Jennings, Z. G., Romanowsky, A. J., Brodie, J. P., et al. 2015, ApJ, 812, L10

Jerjen, H., Binggeli, B., \& Freeman, K. C. 2000, AJ, 119, 593

Karachentsev, I. D., \& Karachentseva, V. E. 2004, Astron. Rep., 48, 267

Karachentsev, I. D., Karachentseva, V. E., \& Huchtmeier, W. K. 2001, A\&A, 366,428

Karachentsev, I. D., Karachentseva, V. E., Huchtmeier, W. K., \& Makarov, D. I. 2004, AJ, 127, 2031

Karachentsev, I. D., Makarov, D. I., \& Kaisina, E. I. 2013, AJ, 145, 101

Kim, D., Jerjen, H., Mackey, D., Da Costa, G. S., \& Milone, A. P. 2015, ApJ, 804, L44

Koda, J., Yagi, M., Yamanoi, H., \& Komiyama, Y. 2015, ApJ, 807, L2

Kraan-Korteweg, R. C., \& Tammann, G. A. 1979, Astron. Nachr., 300, 181

Kroupa, P., Theis, C., \& Boily, C. M. 2005, A\&A, 431, 517

Kroupa, P., Famaey, B., de Boer, K. S., et al. 2010, A\&A, 523, A32

Lisker, T., Grebel, E. K., \& Binggeli, B. 2008, AJ, 135, 380

Lupton, R. 2005, Transformations between SDSS magnitudes and other systems https://www.sdss3.org/dr10/algorithms/sdssUBVRITransform . php/

Makarov, D., \& Karachentsev, I. 2011, MNRAS, 412, 2498

Makarova, L. N., Makarov, D. I., Antipova, A. V., Karachentsev, I. D., \& Tully, R. B. 2018, MNRAS, 474, 3221

McConnachie, A. W. 2012, AJ, 144, 4

Merritt, A., van Dokkum, P., \& Abraham, R. 2014, ApJ, 787, L37

Merritt, A., van Dokkum, P., Danieli, S., et al. 2016, ApJ, 833, 168

Michel-Dansac, L., Duc, P.-A., Bournaud, F., et al. 2010, ApJ, 717, L143

Moore, B., Ghigna, S., Governato, F., et al. 1999, ApJ, 524, L19

Müller, O., Jerjen, H., \& Binggeli, B. 2015, A\&A, 583, A79

Müller, O., Jerjen, H., Pawlowski, M. S., \& Binggeli, B. 2016, A\&A, 595, A119

Müller, O., Jerjen, H., \& Binggeli, B. 2017a, A\&A, 597, A7

Müller, O., Scalera, R., Binggeli, B., \& Jerjen, H. 2017b, A\&A, 602, A119

Müller, O., Pawlowski, M. S., Jerjen, H., \& Lelli, F. 2018, Science, 359, 534

Nataf, D. M. 2015, MNRAS, 449, 1171

Nikiel-Wroczyński, B., Soida, M., Bomans, D. J., \& Urbanik, M. 2014, ApJ, 786, 144

Park, H. S., Moon, D.-S., Zaritsky, D., et al. 2017, ApJ, 848, 19

Pawlowski, M. S. 2018, Mod. Phys. Lett. A, 33, 1830004

Pawlowski, M. S., Pflamm-Altenburg, J., \& Kroupa, P. 2012, MNRAS, 423, 1109

Rekola, R., Jerjen, H., \& Flynn, C. 2005, A\&A, 437, 823

Sandage, A., \& Binggeli, B. 1984, AJ, 89, 919

Schlafly, E. F., \& Finkbeiner, D. P. 2011, ApJ, 737, 103

Schneider, S. 1985, ApJ, 288, L33

Schombert, J. M., Pildis, R. A., \& Eder, J. A. 1997, ApJS, 111, 233

Sersic, J. L. 1968, Atlas de galaxias australes (Córdoba, Argentina: Observatorio Astronómico)

Speller, R., \& Taylor, J. E. 2014, ApJ, 788, 188

Staveley-Smith, L., Davies, R. D., \& Kinman, T. D. 1992, MNRAS, 258, 334

Stierwalt, S., Haynes, M. P., Giovanelli, R., et al. 2009, AJ, 138, 338

Trentham, N., \& Tully, R. B. 2002, MNRAS, 335, 712

Tully, R. B., \& Fisher, J. R. 1988, Catalog of Nearby Galaxies (Cambridge University Press)

Tully, R. B., Courtois, H. M., Dolphin, A. E., et al. 2013, AJ, 146, 86

Tully, R. B., Libeskind, N. I., Karachentsev, I. D., et al. 2015, ApJ, 802, L25

van der Burg, R. F. J., Muzzin, A., \& Hoekstra, H. 2016, A\&A, 590, A20

van Dokkum, P. G., Abraham, R., Merritt, A., et al. 2015, ApJ, 798, L45

Venhola, A., Peletier, R., Laurikainen, E., et al. 2017, A\&A, 608, A142

Watkins, A. E., Mihos, J. C., Harding, P., \& Feldmeier, J. J. 2014, ApJ, 791, 38

Wittmann, C., Lisker, T., Ambachew Tilahun, L., et al. 2017, MNRAS, 470, 1512

Wong, O. I., Ryan-Weber, E. V., Garcia-Appadoo, D. A., et al. 2006, MNRAS, 371,1855

Zwicky, F. 1956, Ergebnisse der exakten Naturwissenschaften, 29, 344 
O. Müller et al.: The Leo-I group: new dwarf galaxy and UDG candidates

\section{Appendix A: Tables}

Table A.1. Names, coordinates, and morphological types of the 36 new dwarf galaxy candidates of the Leo-I group.

\begin{tabular}{|c|c|c|c|c|}
\hline Name & $\begin{array}{c}\alpha \\
(\mathrm{J} 2000) \\
\end{array}$ & $\begin{array}{c}\delta \\
(\mathrm{J} 2000)\end{array}$ & Type & Notes \\
\hline dw1013+18 & $10: 13: 29.0$ & $+18: 36: 44$ & $\mathrm{dIrr} / \mathrm{dSph}$ & field \\
\hline dw1037+09 & $10: 37: 40.7$ & $+09: 06: 20$ & dIrr & M96 \\
\hline dw1040+06 & $10: 40: 30.3$ & $+06: 56: 28$ & $\mathrm{dSph}$ & field \\
\hline dw1044+11 & $10: 44: 33.0$ & $+11: 16: 10$ & $\mathrm{dSph}$ & M96 \\
\hline $\mathrm{dw} 1045+14 \mathrm{a}$ & $10: 45: 00.6$ & $+14: 06: 20$ & $\mathrm{dIrr} / \mathrm{dSph}$ & M96 \\
\hline$d w 1045+14 b$ & $10: 45: 56.3$ & $+14: 13: 37$ & $\mathrm{dSph}$ & M96 \\
\hline dw1045+16 & $10: 45: 56.3$ & $+16: 55: 00$ & dSph, bg? & M96 \\
\hline dw1045+13 & $10: 45: 58.1$ & $+13: 32: 52$ & $\mathrm{dSph}$ & M96 \\
\hline dw1047+16 & $10: 47: 00.0$ & $+16: 08: 50$ & $\mathrm{dSph}, \mathrm{N}$ & M96 \\
\hline dw1048+13 & $10: 48: 35.7$ & $+13: 03: 34$ & $\mathrm{dSph}$ & M96 \\
\hline $\mathrm{dw} 1049+12 \mathrm{a}$ & $10: 49: 11.4$ & $+12: 47: 34$ & $\mathrm{dSph}$ & M96 \\
\hline dw1049+15 & $10: 49: 14.3$ & $+15: 58: 20$ & $\mathrm{dSph} / \mathrm{dIrr}$ & M96 \\
\hline dw1049+12b & $10: 49: 25.8$ & $+12: 33: 08$ & $\mathrm{dSph} / \mathrm{dIrr} ?$ & M96 \\
\hline dw1051+11 & $10: 51: 03.8$ & $+11: 01: 13$ & dSph, UDG? & M96 \\
\hline dw1055+11 & $10: 55: 43.5$ & $+11: 58: 05$ & dSph,N, UDG? & M96 \\
\hline dw1059+11 & $10: 59: 50.9$ & $+11: 25: 38$ & $\mathrm{dSph}$ & M96 \\
\hline dw1101+11 & $11: 01: 22.5$ & $+11: 45: 12$ & $\mathrm{dSph}$ & M96 \\
\hline dw1109+18 & 11:09:08.5 & $+18: 54: 22$ & $\mathrm{dIrr} / \mathrm{dSph}$ & field \\
\hline dw1110+18 & $11: 10: 54.9$ & $+18: 58: 52$ & $\mathrm{dSph}$ & field \\
\hline dw1116+14 & $11: 16: 14.4$ & $+14: 38: 21$ & dSph, bg? & Leo-Tr \\
\hline dw1116+15a & $11: 16: 17.1$ & $+15: 04: 02$ & dSph, bg? & Leo-Tr \\
\hline$d w 1116+15 b$ & $11: 16: 46.4$ & $+15: 54: 19$ & $\mathrm{dIrr} / \mathrm{dSph}, \mathrm{bg} ?$ & Leo-Tr \\
\hline dw1117+15 & $11: 17: 02.1$ & $+15: 10: 17$ & dSph, UDG?, bg? & Leo- $\operatorname{Tr}$ \\
\hline dw1117+12 & $11: 17: 44.2$ & $+12: 50: 10$ & $\mathrm{dSph}$ & Leo-Tr \\
\hline dw1118+13a & $11: 18: 15.9$ & $+13: 30: 53$ & $\mathrm{dSph}$ & Leo-Tr \\
\hline$d w 1118+13 b$ & $11: 18: 53.3$ & $+13: 48: 18$ & $\mathrm{dSph}$ & Leo-Tr \\
\hline dw1123+13 & $11: 23: 56.4$ & $+13: 46: 41$ & $\mathrm{dSph}$ & Leo-Tr \\
\hline dw $1127+13$ & $11: 27: 13.0$ & $+13: 46: 50$ & $\mathrm{dSph}$ & Leo-Tr \\
\hline dw1130+20 & $11: 30: 32.0$ & $+20: 45: 41$ & dIrr & field \\
\hline dw1131+15 & $11: 31: 01.0$ & $+15: 54: 52$ & $\mathrm{dSph}$ & field \\
\hline dw1137+16 & $11: 37: 45.6$ & $+16: 31: 09$ & dSph, UDG? & field \\
\hline dw1140+17 & $11: 40: 43.0$ & $+17: 38: 33$ & $\mathrm{dSph}$ & field \\
\hline dw1145+14 & $11: 45: 32.1$ & $+15: 52: 50$ & $\mathrm{dSph}$ & field \\
\hline dw1148+12 & 11:48:09.1 & $+12: 48: 47$ & $\mathrm{dSph}$ & field \\
\hline dw1148+16 & $11: 48: 45.0$ & $+16: 44: 24$ & $\mathrm{dIrr} / \mathrm{dSph}$ & field \\
\hline dw1151+16 & $11: 51: 15.2$ & $+16: 00: 20$ & $\mathrm{dSph}$ & field \\
\hline
\end{tabular}


Table A.2. Photometric and structural parameters of the new dwarf candidates in the surveyed region of the Leo-I group.

\begin{tabular}{|c|c|c|c|c|c|c|c|c|c|c|c|c|}
\hline Name & $\begin{array}{r}g_{\text {tot }} \\
\text { mag }\end{array}$ & $\begin{array}{l}r_{\text {tot }} \\
\text { mag }\end{array}$ & $\begin{array}{r}A_{g} \\
\text { mag }\end{array}$ & $\begin{array}{r}A_{r} \\
\text { mag }\end{array}$ & $\begin{array}{r}M_{r} \\
\text { mag }\end{array}$ & $\begin{array}{r}(g-r)_{0, \text { tot }} \\
\text { mag }\end{array}$ & $\begin{array}{c}\mu_{0, r} \\
\operatorname{mag} \operatorname{arcsec}^{-2}\end{array}$ & $\begin{array}{r}r_{0, r} \\
\operatorname{arcsec}\end{array}$ & $n_{r}$ & $\begin{array}{c}\langle\mu\rangle_{\mathrm{eff}, r} \\
\operatorname{mag} \operatorname{arcsec}^{-2}\end{array}$ & $\begin{array}{r}r_{\mathrm{eff}, \mathrm{r}} \\
\operatorname{arcsec}\end{array}$ & $\begin{array}{r}\log r_{\mathrm{eff}, \mathrm{t}} \\
\log \mathrm{pc}\end{array}$ \\
\hline (1) & (2) & (3) & (4) & (5) & (6) & (7) & (8) & (9) & (10) & (11) & (12) & (13) \\
\hline
\end{tabular}

\begin{tabular}{|c|c|c|c|c|c|c|c|c|c|c|c|c|}
\hline $\begin{array}{l}\text { M96 subgroup } \\
\text { dw } 1037+09\end{array}$ & 17.61 & 17.09 & 0.080 & 0.056 & -13.04 & 0.496 & $24.04 \pm 0.05$ & $9.96 \pm 0.38$ & $1.68 \pm 0.14$ & 25.44 & 18.7 & 2.97 \\
\hline dw1044+11 & 19.39 & 19.17 & 0.088 & 0.061 & -10.97 & 0.200 & $25.42 \pm 0.45$ & $10.87 \pm 5.89$ & $0.87 \pm 0.40$ & 26.59 & 12.1 & 2.78 \\
\hline $\mathrm{dw} 1045+14 \mathrm{a}$ & 19.02 & 18.68 & 0.097 & 0.067 & -11.46 & 0.313 & $22.79 \pm 0.63$ & $1.25 \pm 1.14$ & $0.56 \pm 0.16$ & 24.87 & 6.92 & 2.54 \\
\hline $\mathrm{dw} 1045+14 \mathrm{~b}$ & 19.79 & 19.29 & 0.094 & 0.065 & -10.85 & 0.470 & $24.50 \pm 0.17$ & $5.24 \pm 0.83$ & $1.13 \pm 0.18$ & 25.39 & 6.61 & 2.52 \\
\hline $\mathrm{dw} 1045+16$ & 18.50 & 17.62 & 0.085 & 0.059 & -12.51 & 0.854 & $24.59 \pm 0.30$ & $8.39 \pm 3.22$ & $0.81 \pm 0.24$ & 26.43 & 23.0 & 3.06 \\
\hline $\mathrm{dw} 1045+13$ & 18.81 & 18.08 & 0.110 & 0.076 & -12.07 & 0.696 & $24.96 \pm 0.15$ & $7.41 \pm 0.70$ & $1.92 \pm 0.52$ & 26.59 & 20.0 & 3.00 \\
\hline $\mathrm{dw} 1047+16$ & 18.07 & 17.91 & 0.091 & 0.063 & -12.23 & 0.128 & $22.77 \pm 0.55$ & $1.32 \pm 1.18$ & $0.49 \pm 0.13$ & 25.17 & 11.2 & 2.75 \\
\hline $\mathrm{dw} 1048+13$ & 19.83 & 18.67 & 0.111 & 0.077 & -11.48 & 1.126 & $25.53 \pm 0.10$ & $13.63 \pm 0.64$ & $2.94 \pm 0.78$ & 26.19 & 12.6 & 2.80 \\
\hline $\mathrm{dw} 1049+12 \mathrm{a}$ & 19.39 & 18.98 & 0.088 & 0.061 & -11.16 & 0.386 & $23.78 \pm 0.49$ & $2.92 \pm 1.91$ & $0.67 \pm 0.19$ & 25.54 & 8.18 & 2.61 \\
\hline $\mathrm{dw} 1049+15$ & 18.56 & 17.88 & 0.088 & 0.061 & -12.26 & 0.655 & $24.30 \pm 0.12$ & $9.38 \pm 0.90$ & $1.42 \pm 0.23$ & 25.08 & 10.9 & 2.74 \\
\hline $\mathrm{dw} 1049+12 \mathrm{~b}$ & 19.10 & 18.05 & 0.085 & 0.059 & -12.08 & 1.020 & $24.74 \pm 0.28$ & $9.76 \pm 3.03$ & $0.96 \pm 0.36$ & 26.04 & 15.7 & 2.90 \\
\hline $\mathrm{dw} 1051+11$ & 17.85 & 16.95 & 0.092 & 0.063 & -13.19 & 0.872 & $25.34 \pm 0.07$ & $16.76 \pm 0.63$ & $4.15 \pm 1.20$ & 26.20 & 28.2 & 3.15 \\
\hline dw1055+11 & 17.59 & 16.40 & 0.066 & 0.046 & -13.72 & 1.169 & $24.88 \pm 0.28$ & $18.86 \pm 3.90$ & $0.97 \pm 0.54$ & 26.18 & 36.0 & 3.25 \\
\hline $\mathrm{dw} 1059+11$ & 18.98 & 18.60 & 0.060 & 0.041 & -11.51 & 0.359 & $24.61 \pm 0.11$ & $9.73 \pm 0.72$ & $1.68 \pm 0.26$ & 25.02 & 7.65 & 2.58 \\
\hline dw1101+11 & 19.47 & 19.45 & 0.058 & 0.040 & -10.66 & 0.005 & $23.33 \pm 1.62$ & $1.16 \pm 2.78$ & $0.50 \pm 0.32$ & 25.56 & 6.64 & 2.52 \\
\hline \multicolumn{13}{|l|}{ Leo Triplet } \\
\hline dw1116+14 & 20.33 & 19.57 & 0.071 & 0.049 & -10.56 & 0.742 & $25.67 \pm 0.13$ & $10.63 \pm 0.58$ & $3.28 \pm 1.11$ & 25.81 & 7.08 & 2.55 \\
\hline dw1116+15a & 20.26 & 19.80 & 0.076 & 0.052 & -10.33 & 0.437 & $25.11 \pm 0.32$ & $6.79 \pm 2.43$ & $0.95 \pm 0.28$ & 25.98 & 6.88 & 2.54 \\
\hline$d w 1116+15 b$ & 20.42 & 19.31 & 0.068 & 0.047 & -10.81 & 1.091 & $25.33 \pm 0.26$ & $8.85 \pm 2.61$ & $1.00 \pm 0.32$ & 27.02 & 13.8 & 2.84 \\
\hline dw1117+15 & 17.56 & 17.25 & 0.082 & 0.057 & -12.88 & 0.280 & $25.57 \pm 0.07$ & $17.11 \pm 0.51$ & $3.79 \pm 0.92$ & 27.31 & 40.9 & 3.31 \\
\hline dw1117+12 & 21.22 & 19.87 & 0.073 & 0.050 & -10.25 & 1.322 & $25.24 \pm 0.52$ & $7.29 \pm 4.29$ & $0.93 \pm 0.51$ & 26.10 & 7.02 & 2.54 \\
\hline $\mathrm{dw} 1118+13 \mathrm{a}$ & 19.49 & 19.59 & 0.077 & 0.053 & -10.54 & -0.11 & $25.88 \pm 0.24$ & $14.36 \pm 1.94$ & $1.74 \pm 1.02$ & 26.36 & 9.04 & 2.65 \\
\hline $\mathrm{dw} 1118+13 \mathrm{~b}$ & 18.15 & 17.78 & 0.069 & 0.047 & -12.34 & 0.341 & $25.33 \pm 0.13$ & $15.09 \pm 1.66$ & $1.35 \pm 0.24$ & 26.45 & 21.5 & 3.03 \\
\hline dw1123+13 & 19.62 & 19.08 & 0.079 & 0.054 & -11.05 & 0.513 & $24.95 \pm 0.16$ & $8.74 \pm 1.05$ & $1.55 \pm 0.31$ & 25.38 & 7.26 & 2.56 \\
\hline $\mathrm{dw} 1127+13$ & 19.76 & 18.85 & 0.093 & 0.064 & -11.28 & 0.872 & $25.51 \pm 0.13$ & $12.85 \pm 0.95$ & $1.72 \pm 0.52$ & 26.10 & 11.2 & 2.75 \\
\hline \multicolumn{13}{|l|}{ Field } \\
\hline $\mathrm{dw} 1013+18$ & 18.02 & 17.65 & 0.106 & 0.073 & -12.50 & 0.340 & $22.67 \pm 0.10$ & $3.29 \pm 0.38$ & $0.76 \pm 0.04$ & 24.16 & 7.99 & 2.60 \\
\hline dw1040+06 & 17.96 & 18.22 & 0.120 & 0.083 & -11.94 & -0.29 & $24.36 \pm 0.12$ & $8.18 \pm 0.88$ & $1.20 \pm 0.17$ & 25.37 & 10.7 & 2.73 \\
\hline dw1109+18 & 17.73 & 17.18 & 0.077 & 0.054 & -12.95 & 0.523 & $23.25 \pm 0.06$ & $7.35 \pm 0.41$ & $1.07 \pm 0.06$ & 24.17 & 9.94 & 2.70 \\
\hline dw1110+18 & 18.00 & 17.39 & 0.077 & 0.053 & -12.74 & 0.587 & $24.30 \pm 0.11$ & $12.15 \pm 1.16$ & $1.20 \pm 0.20$ & 25.15 & 14.2 & 2.85 \\
\hline dw1130+20 & 17.53 & 17.28 & 0.068 & 0.047 & -12.84 & 0.220 & $22.63 \pm 0.14$ & $2.75 \pm 0.58$ & $0.61 \pm 0.05$ & 24.49 & 10.9 & 2.74 \\
\hline dw1131+15 & 19.51 & 18.87 & 0.171 & 0.118 & -11.32 & 0.581 & $24.59 \pm 0.09$ & $7.39 \pm 0.42$ & $1.97 \pm 0.24$ & 25.22 & 7.43 & 2.57 \\
\hline dw1137+16 & 17.32 & 16.77 & 0.097 & 0.067 & -13.37 & 0.523 & $24.49 \pm 0.12$ & $14.19 \pm 1.99$ & $0.89 \pm 0.10$ & 25.89 & 26.6 & 3.12 \\
\hline dw1140+17 & 18.54 & 17.89 & 0.098 & 0.068 & -12.25 & 0.623 & $24.85 \pm 0.20$ & $13.96 \pm 3.30$ & $0.87 \pm 0.23$ & 25.67 & 14.3 & 2.85 \\
\hline dw1145+14 & 19.86 & 19.20 & 0.147 & 0.101 & -10.97 & 0.613 & $24.02 \pm 0.12$ & $4.94 \pm 0.49$ & $1.39 \pm 0.17$ & 24.65 & 4.89 & 2.39 \\
\hline dw1148+12 & 17.95 & 17.91 & 0.119 & 0.082 & -12.25 & 0.002 & $24.58 \pm 0.20$ & $9.02 \pm 1.75$ & $1.02 \pm 0.29$ & 25.78 & 14.9 & 2.87 \\
\hline dw1148+16 & 17.49 & 17.34 & 0.150 & 0.104 & -12.84 & 0.109 & $22.85 \pm 0.06$ & $3.77 \pm 0.32$ & $0.78 \pm 0.04$ & 24.61 & 11.3 & 2.75 \\
\hline dw1151+16 & 18.04 & 18.27 & 0.109 & 0.075 & -11.88 & -0.25 & $22.92 \pm 0.07$ & $3.92 \pm 0.25$ & $1.09 \pm 0.05$ & 23.86 & 5.24 & 2.42 \\
\hline
\end{tabular}

Notes. The quantities listed are as follows: (1) name of candidate; (2-3) total apparent magnitude in the $g$ and $r$ bands; (4-5) galactic extinction in the $g$ and $r$ bands (Schlafly \& Finkbeiner 2011); (6) extinction corrected absolute $r$ band magnitude, using a distance modulus of $M-m=30.06$ mag; (7) integrated and extinction corrected $g-r$ color; (8) Sérsic central surface brightness in the $r$ band; (9) Sérsic scale length in the $r$ band; (10) Sérsic curvature index in the $r$ band; (11) mean effective surface brightness in the $r$ band; (12) effective radius in the $r$ band; (13) the logarithm of the effective radius in the $r$ band, converted to $\mathrm{pc}$ with a distance modulus of $M-m=30.06$ mag. We note that surface brightness values presented are not extinction corrected. 
O. Müller et al.: The Leo-I group: new dwarf galaxy and UDG candidates

Table A.3. Coordinates of the possible background dwarf galaxy in our survey footprint around bright host galaxies with $v<2000 \mathrm{~km} \mathrm{~s}^{2}$.

\begin{tabular}{|c|c|c|}
\hline Name & $\begin{array}{c}\alpha \\
(\mathrm{J} 2000) \\
\end{array}$ & $\begin{array}{c}\delta \\
(\mathrm{J} 2000) \\
\end{array}$ \\
\hline NGC 3227_1 & $10: 22: 53$ & $+19: 34: 36$ \\
\hline NGC 3227_2 & $10: 24: 43$ & $+19: 57: 16$ \\
\hline NGC 3227_3 & $10: 25: 50$ & $+19: 43: 22$ \\
\hline NGC 3666_1 & $: 24: 45$ & $+11: 20: 04$ \\
\hline NGC 3666_2 & $11: 24: 10$ & $+11: 25: 12$ \\
\hline NGC 3370_1 & $10: 46: 47$ & $+17: 16: 18$ \\
\hline NGC 3607_1 & $11: 14: 22$ & $+18: 02: 38$ \\
\hline NGC 3607_2 & $11: 14$ & $+18: 22: 30$ \\
\hline NGC 3607_3 & $11: 1$ & $+18: 25: 21$ \\
\hline NGC 3607_4 & $11: 15: 36$ & $+18: 01: 04$ \\
\hline NGC 3607_5 & $1: 15$ & $+18: 04: 40$ \\
\hline NGC 3607_6 & $11: 1$ & $+17: 54: 04$ \\
\hline NGC 3607_7 & $11: 1$ & $+17: 56: 25$ \\
\hline NGC 3607_8 & 11:16:11 & $+17: 57: 04$ \\
\hline NGC 3607_9 & $11: 16: 18$ & $+18: 35: 39$ \\
\hline NGC 3607_10 & $11: 10$ & $+18: 11: 35$ \\
\hline NGC 3607_11 & $11: 16: 30$ & $+18: 19: 27$ \\
\hline NGC 3607_12 & $11: 17$ & $+18: 18: 07$ \\
\hline NGC 3607_13 & $11: 1^{\top}$ & $+17: 19: 09$ \\
\hline NGC 3607_14 & $11: 17: 16$ & $+18: 46: 27$ \\
\hline NGC 3607_15 & $11 \cdot 17 \cdot 22$ & $+17: 59: 50$ \\
\hline NGC 3607_16 & $11: 18: 21$ & $+17: 41: 50$ \\
\hline NGC 3607_17 & $11: 19: 13$ & $+18: 05: 47$ \\
\hline NGC 3607_18 & & $+17: 32: 09$ \\
\hline NGC 3607_19 & $11: 24: 08$ & $+18: 13: 16$ \\
\hline NGC 3607_20 & 11:31:01 & $+15: 54: 48$ \\
\hline
\end{tabular}




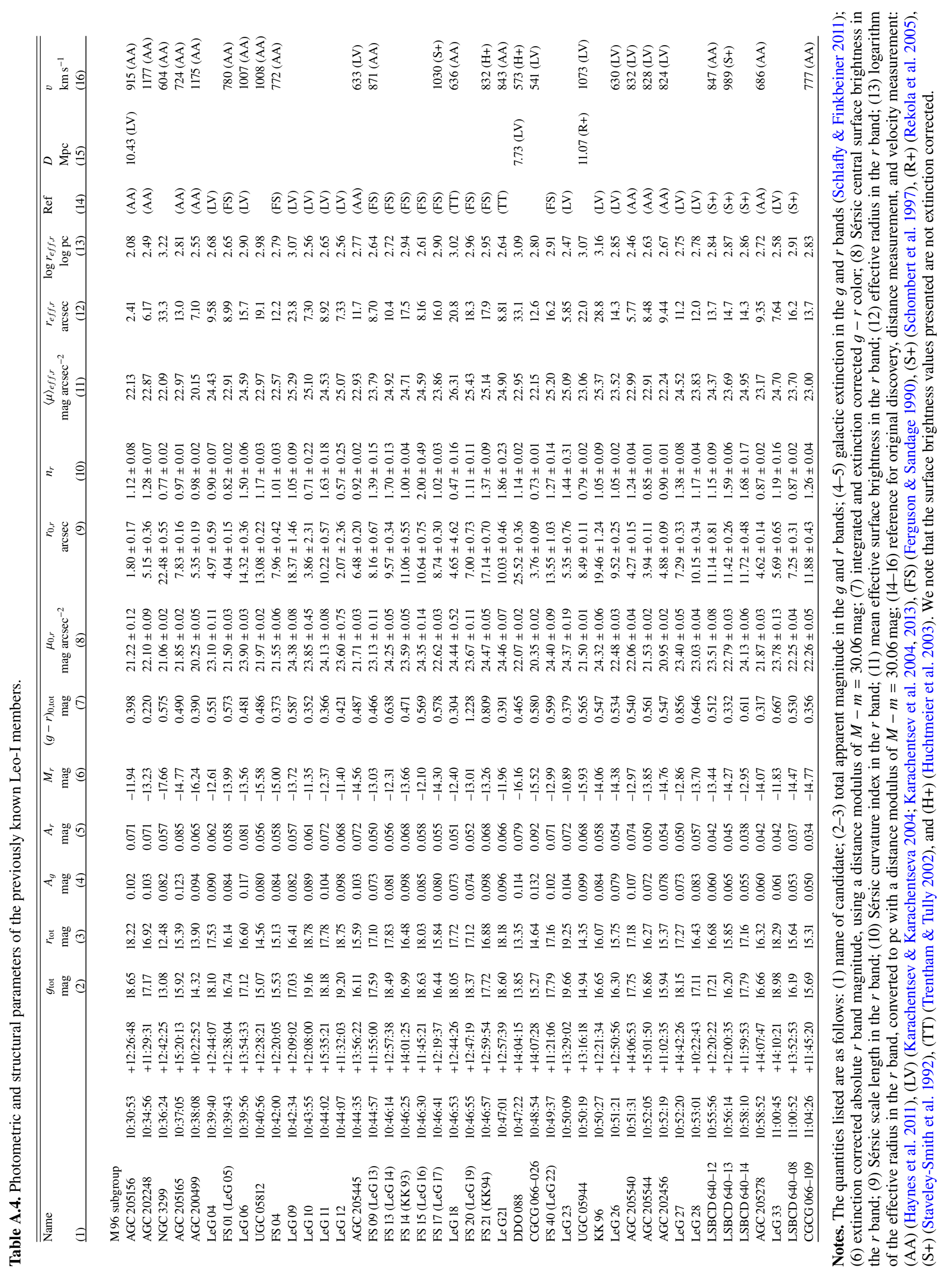

A105, page 12 of 13 


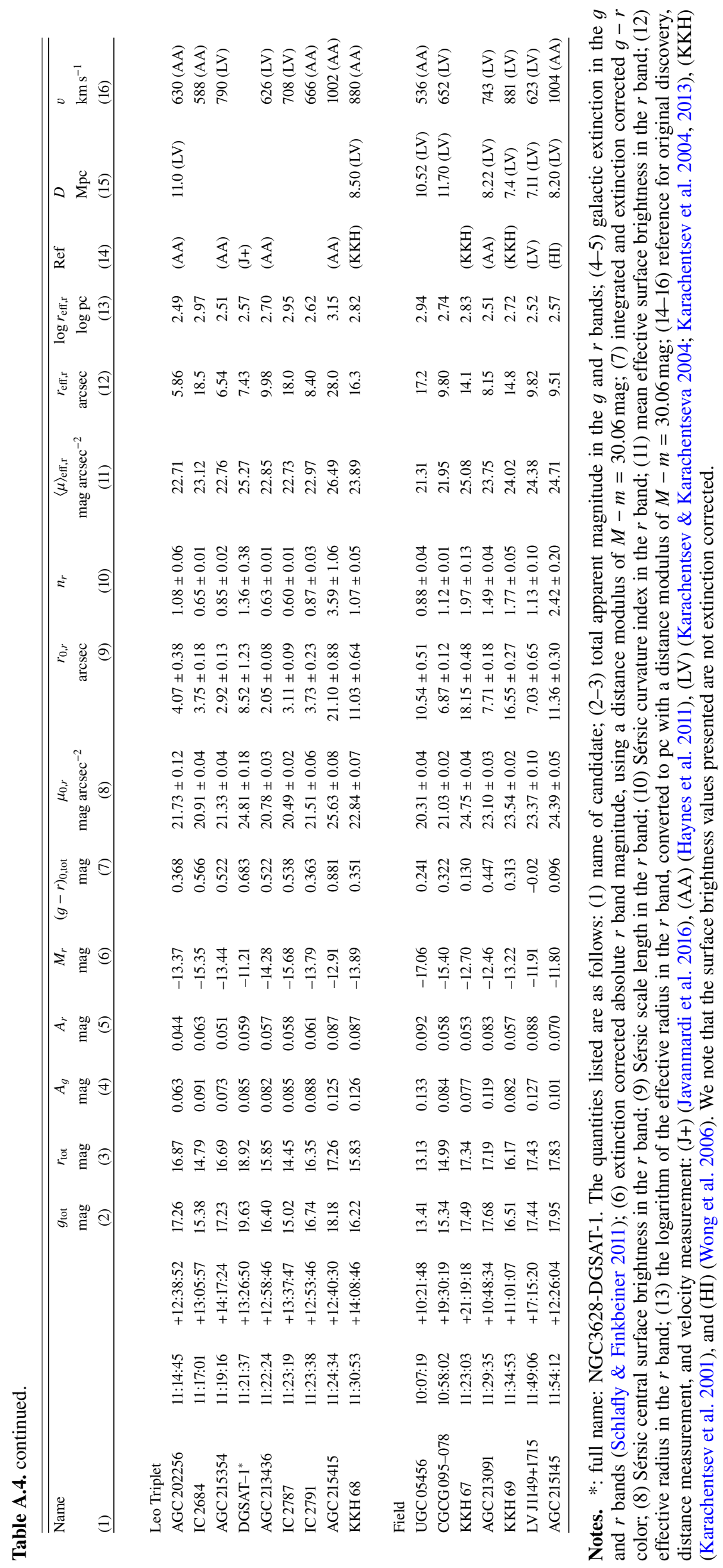

